\title{
Tungsten Experiences in \\ ASDEX Upgrade and JET
}

R. Neu ${ }^{1,2}$ S. Brezinsek 3 , M. Beurskens ${ }^{4}$, V. Bobkov ${ }^{2}$, P. de Vries $^{5}$, C. Giroud $^{4}$, E. Joffrin 6 , A. Kallenbach ${ }^{2}$, G.F. Matthews ${ }^{4}$, M.-L. Mayoral'1,4, G. Pautasso $^{2}$, Th. Pütterich ${ }^{2}$, F. Ryter ${ }^{2}$, J. Schweinzer ${ }^{2}$ and ASDEX Upgrade Team and JET EFDA Contributors

${ }^{1}$ EFDA CSU, Boltzmannstr. 2, 85748 Garching, Germany 2IPP, EURATOM Association, Boltzmannstr. 2, 85748 Garching, Germany 3IEK-4,Association EURATOM/Forschungszentrum Jülich GmbH 52425, Germany ${ }^{4}$ Euratom/CCFE Fusion Association, Culham Science Centre, Abingdon, OX14 3DB, UK ${ }^{5}$ Association EURATOM/DIFFER, Rijnhuizen P0 Box 1207 3430BE Nieuwegen, Netherlands ${ }^{6}$ IRFM-CEA, Centre de Cadarache, 13108 Saint-Paul-lez-Durance, France 


\section{Metal Walls in Support of ITER and DEMO}

\section{AUG \& JET: All Metal PFCs}

- Demonstrate low fuel retention, migration and possible fuel recovery

- Demonstrate plasma compatibility with metallic walls

- Develop tools for improved plasma control and heat load mitigation

- Provide input to the decision on the first ITER divertor (and DEMO main chamber) 


\section{Outline}

- All Metal Walls in AUG and JET

- Operational Experience

- Plasma Breakdown

- Disruptions

- Fuel Retention

-W Behaviour and H-Mode Properties

- W Sources

- W Transport

- LH-Threshold

- Confinement Properties

- Impurity Seeding

- Conclusions 


\section{Full W ASDEX Upgrade}
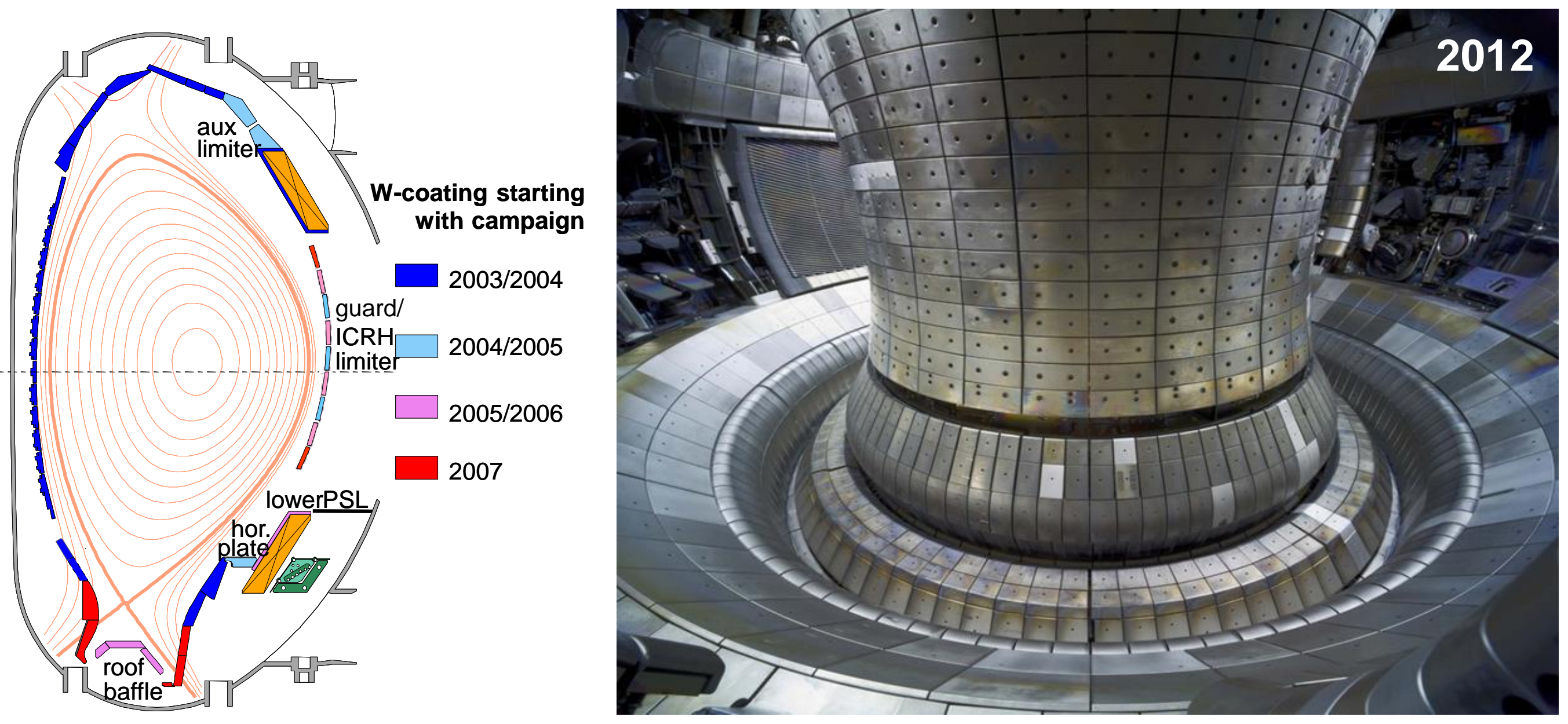

\section{W coatings (3-10 $\mu \mathrm{m})$ on fine grain graphite}




\section{Beryllium}

W-coated CFC Inconel+8 $\mu \mathrm{m}$ Be Bulk W

Poloidal Limiters

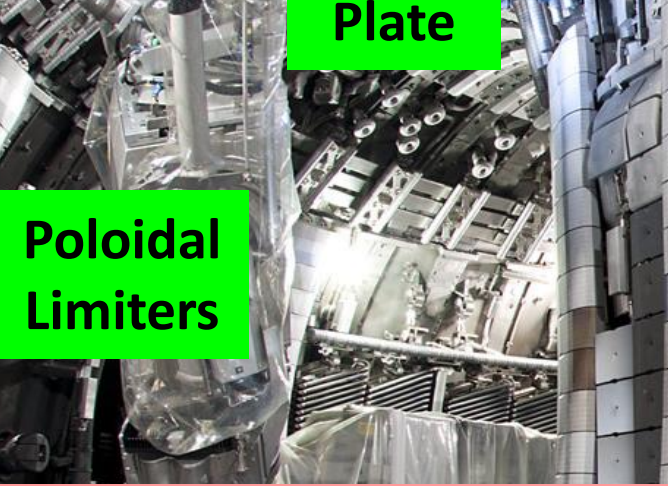

1) foy

ITER materials combination

$\Rightarrow$ 'ITER-like Wall'

(start August 2011)

\section{Additionally:}

- Increased NB power (34MW)

- Improved control tools

- Upgraded diagnostic

Restraint Ring

Protections
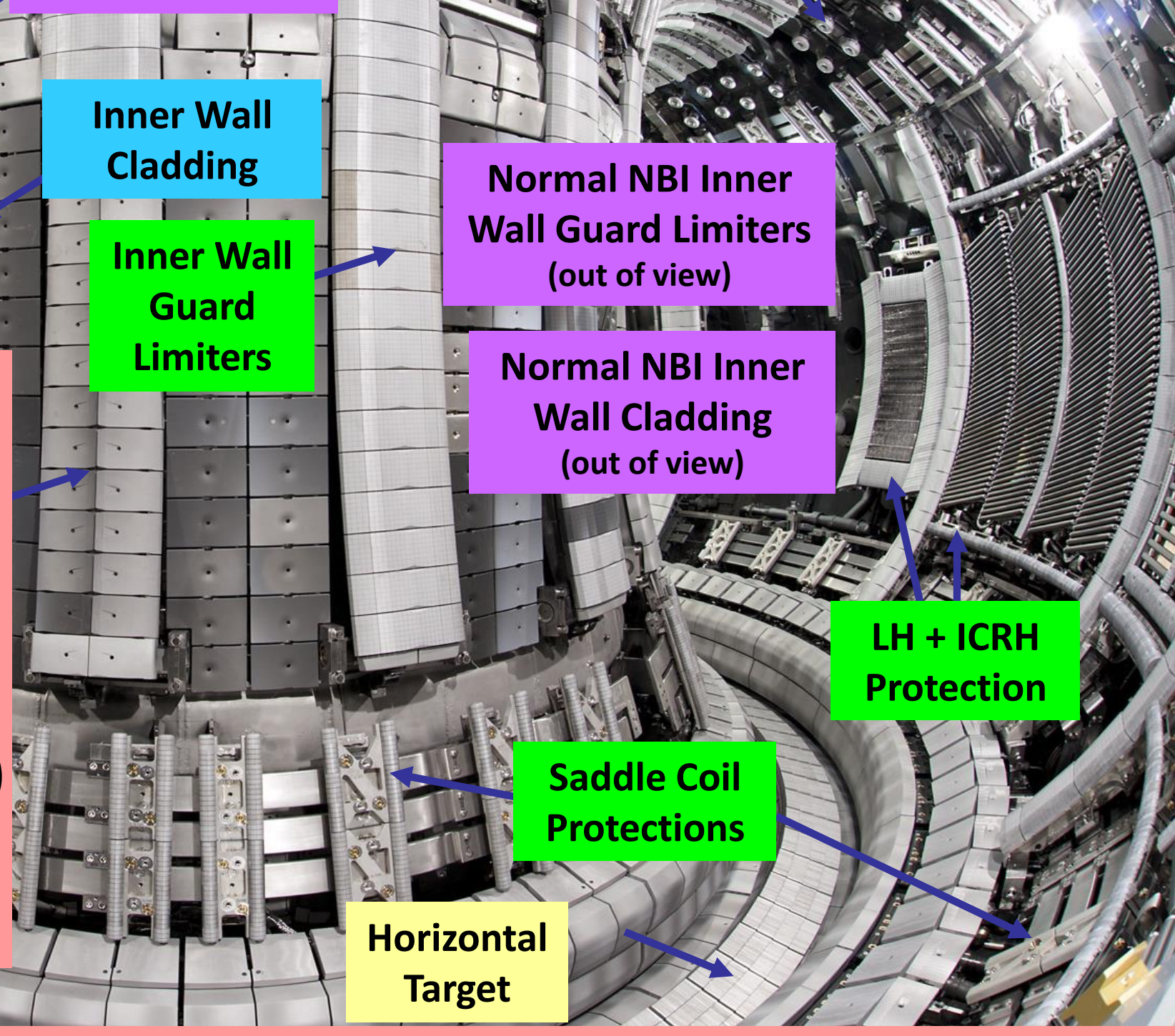

Target
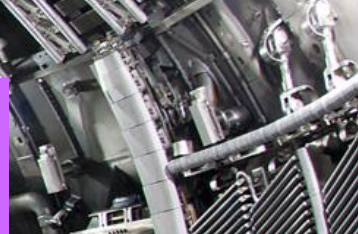

플
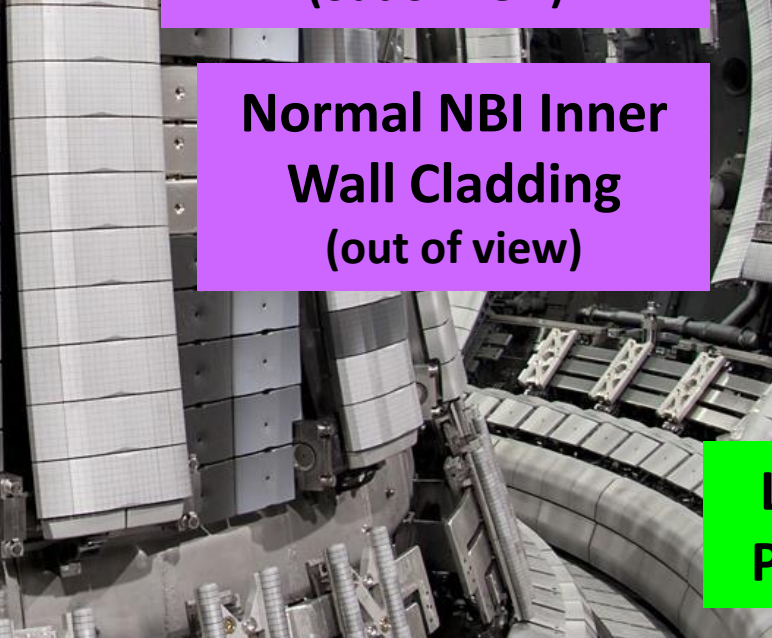
(out of view)
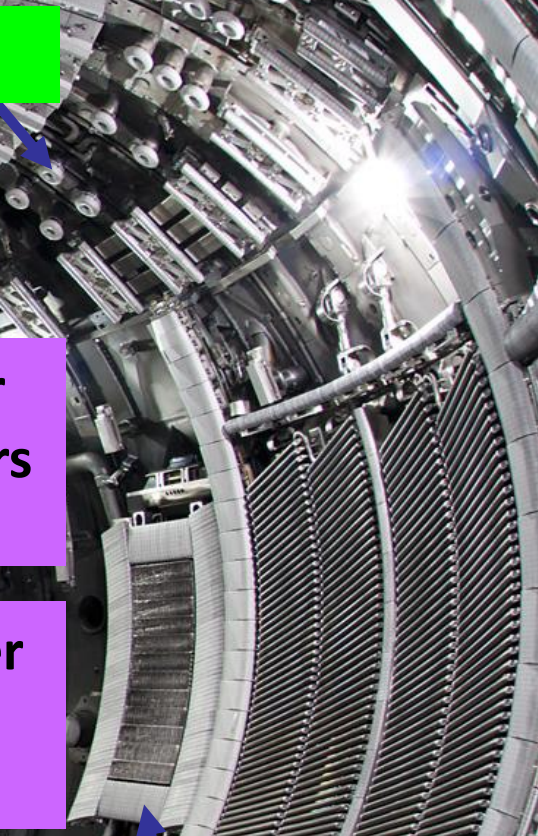

Saddle Coil

Protection

1? H:

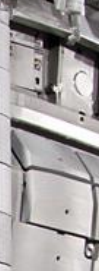

Mushrooms

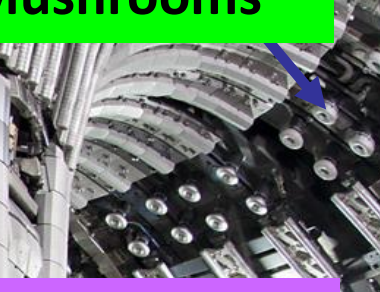

Normal NBI Inner

Wall Guard Limiters (out of view)

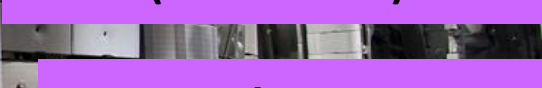

- Normal NBI Inner Wall Cladding (out of view) 


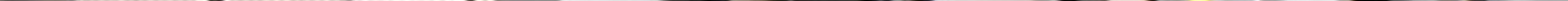




\section{Metal PFCs allow more robust plasma breakdown}

\section{JET ILW}

$1 \mathrm{MA} / 15 \mathrm{~s}$ plasmas established at the first attempt during the 2011 restart

- lower radiation level at higher density (except after $\mathrm{N}_{2}$ seeding) making the breakdown more robust

- unlike the C-wall, no de-conditioning following disruptions (even when using massive gas injection)

$\Rightarrow$ no need for GDC or Be evaporation during operation

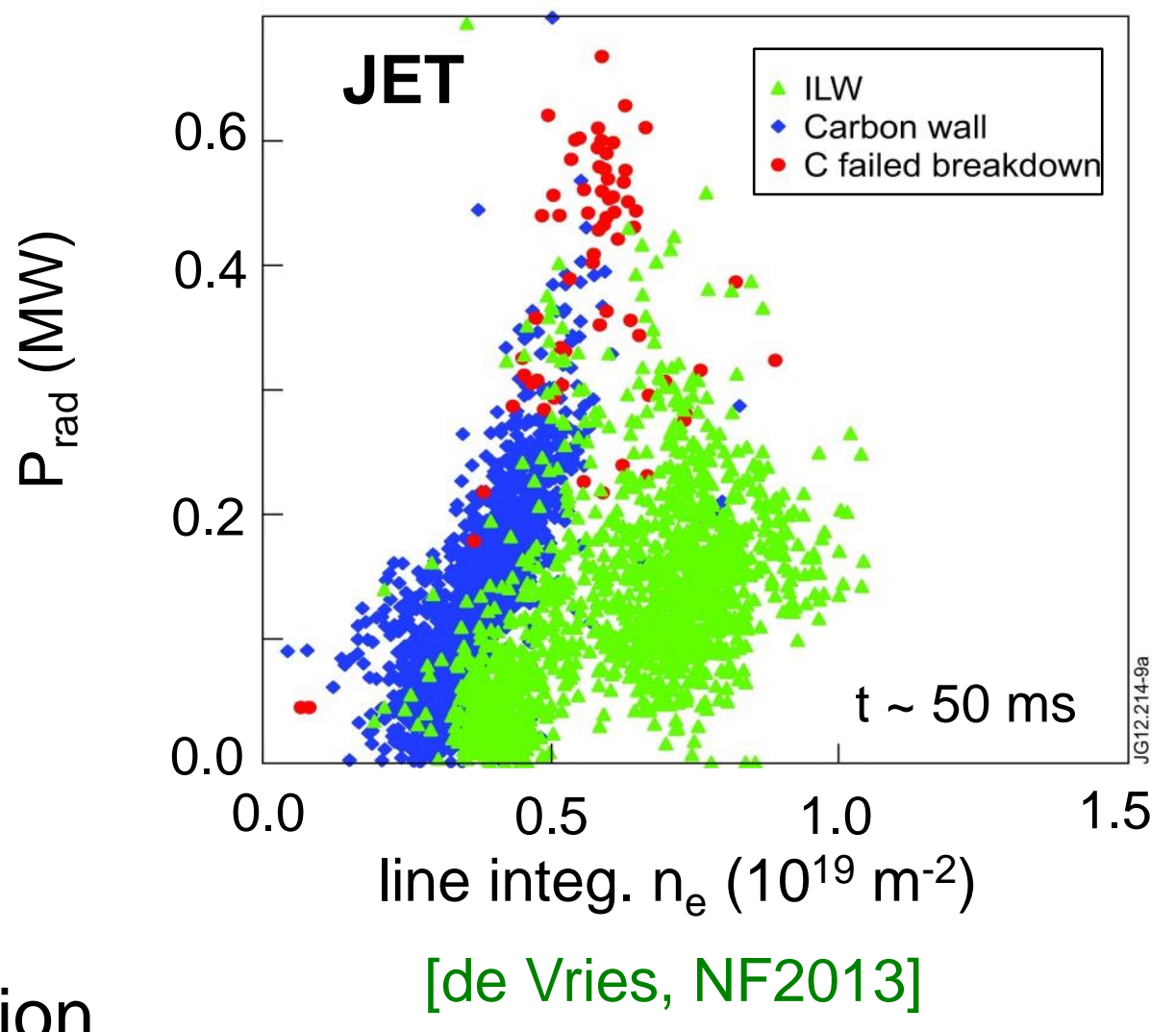

- facilitated start-up even without boronisation

- no need for GDC during normal operation 


\section{Disruption Dynamics Completely Changed}

1200
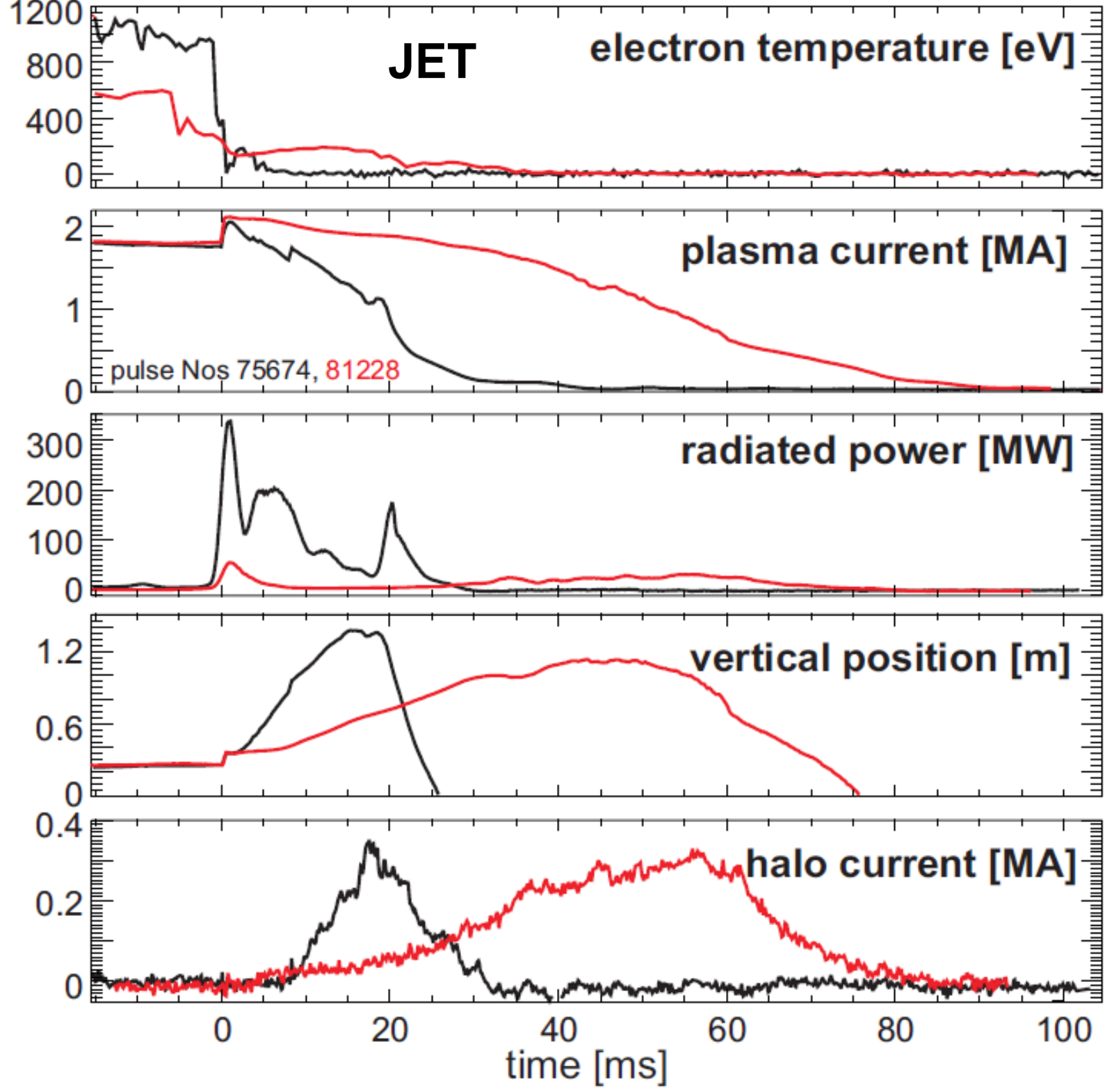

\section{ITER-like wall} hot CQ plasma

slow current decay

low radiation several 10MW only

slower vertical displacement 


\section{Massive Gas Injection Required to Mitigate Disruptions}

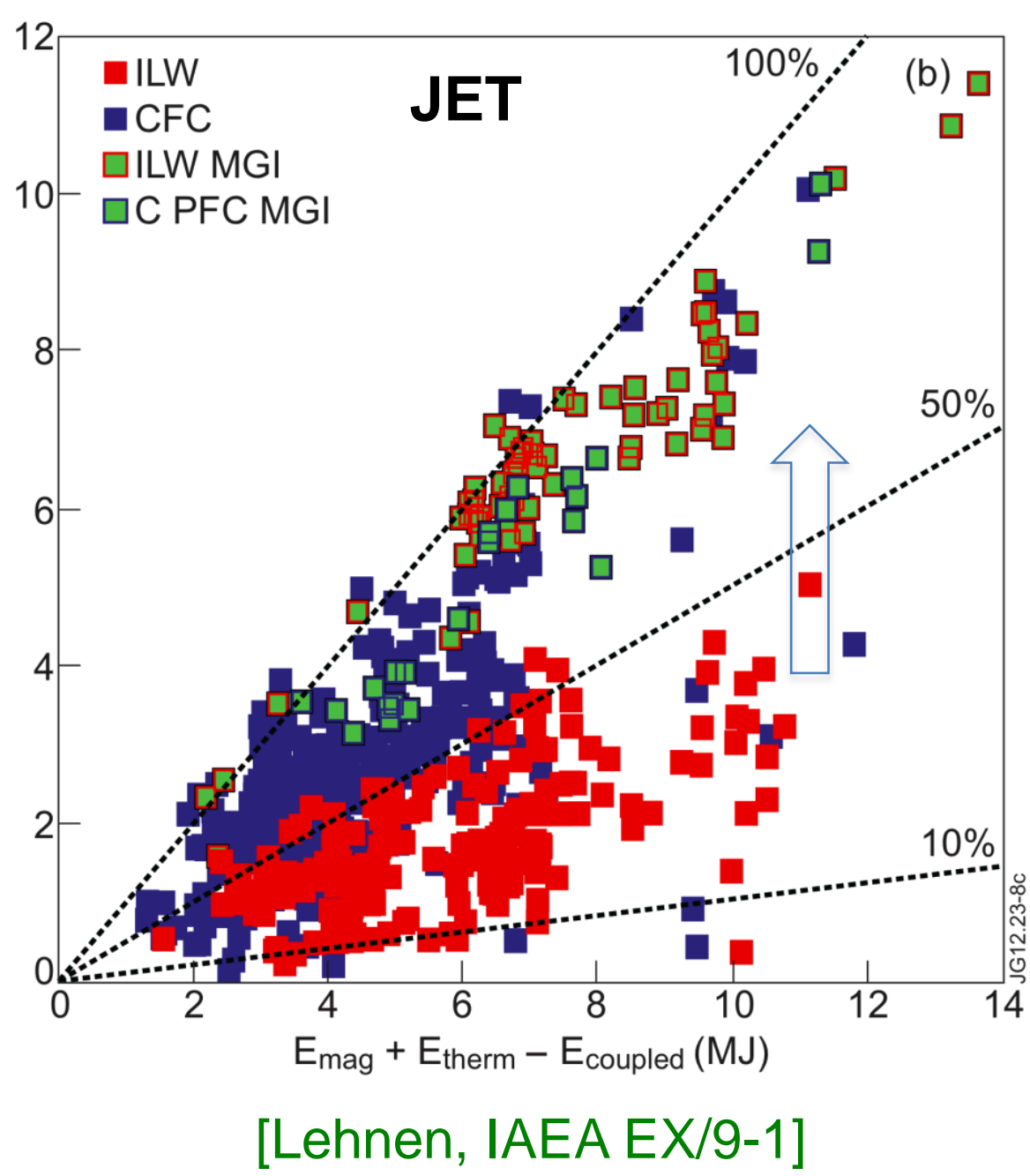

JET-ILW

- massive gas injection $\left(D_{2}+A r\right)$ for disruption mitigation mandatory for $\mathrm{Ip} \geq 2.5 \mathrm{MA}$

- mitigated disruptions with ILW: forces and power loads are return to the level observed with $C$ wall

\section{AUG:}

- similar behaviour but less pronounced

- disruption mitigation is standard procedure 


\section{Fuel Retention in Line With ITER Requirements}

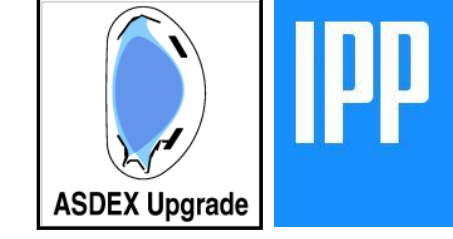

\section{JET ILW}

- global gas balances show reduced retention (factor 10) in all scenarios

- co-deposition with Be should dominate

- even larger reduction expected from "long term outgasing"

$\Rightarrow$ both qualitatively confirmed by first post mortem analysis

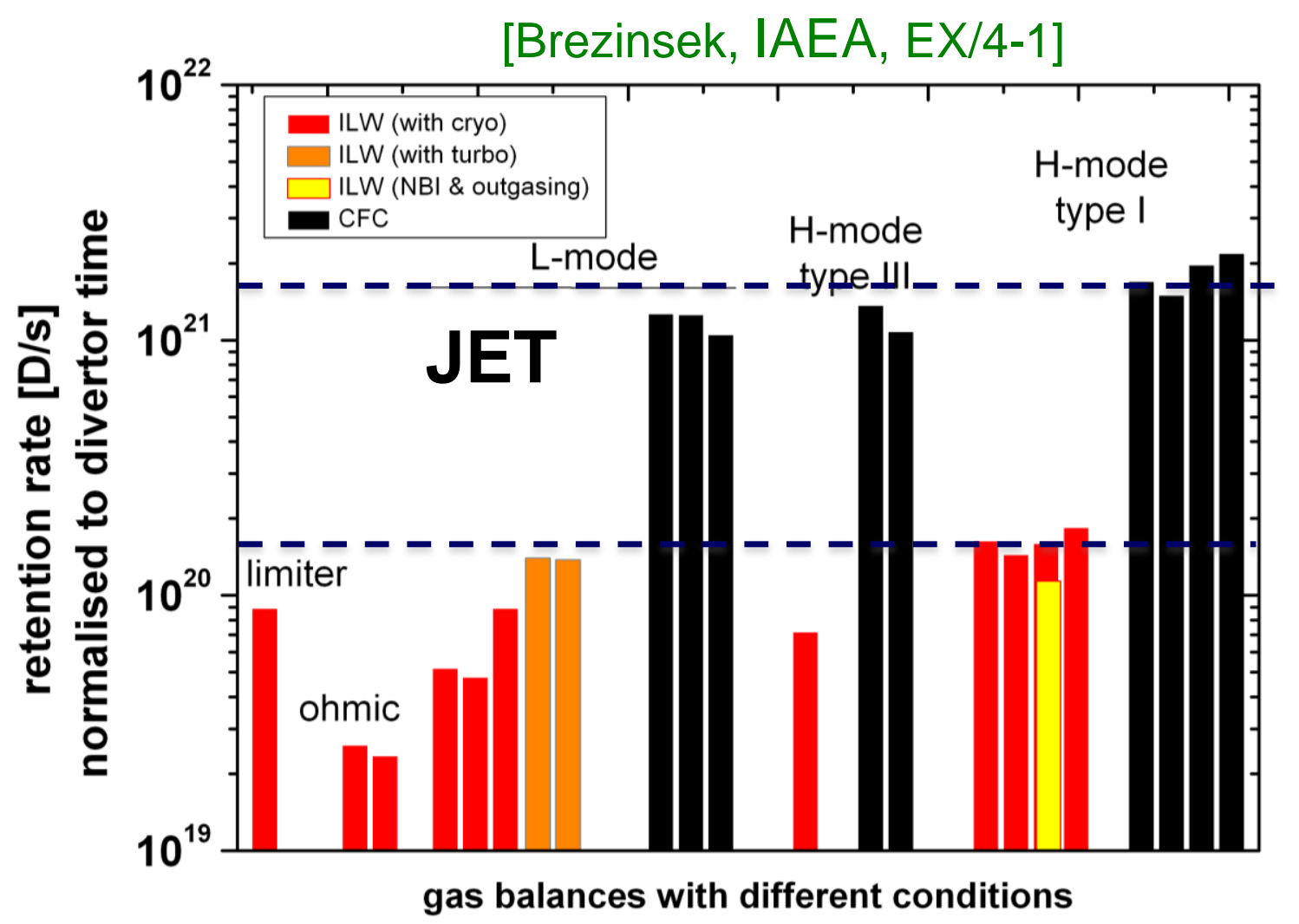

\section{AUG}

- reduction by factor 5-10, dominated by co-deposits with residual $C$ and $B$

- H-retention in W bulk close to values from laboratory experiments

- retention in blisters low in technical W surfaces 


\section{W sources}

$4 \% \mathrm{C}^{4+}$

$3 \% \mathrm{C}^{4+}$

$2 \% \mathrm{C}^{4+}$

$0.5 \% \mathrm{Be}^{2+}$

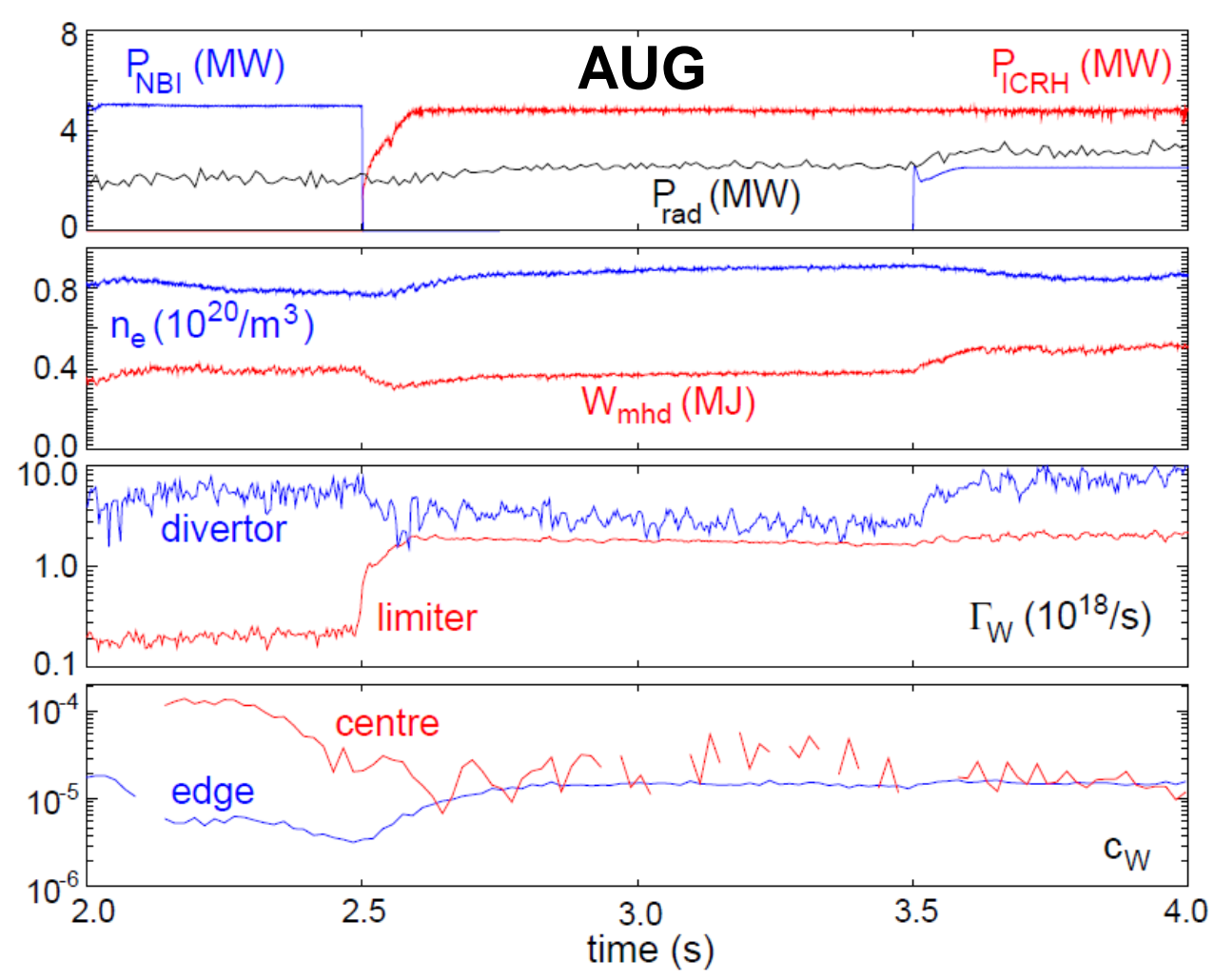

- ICRH strongly increases $\mathrm{W}$ influxes

- AUG: mostly limiter source

- JET: unidentified main chamber source

- increased W sputtering by rectified sheath 


\section{Control of W transport}

- central deposition of heating power suppresses W peaking

- NBI only heated discharges usually show peaked W profile

- ICRH \& ECRH mitigate central accumulation

(critical power

density needed)

- additional W-influx

during ICRH may

outweigh beneficial

effect on central transport

- pellet ELM pace-making helps to keep edge $c_{w}$ low

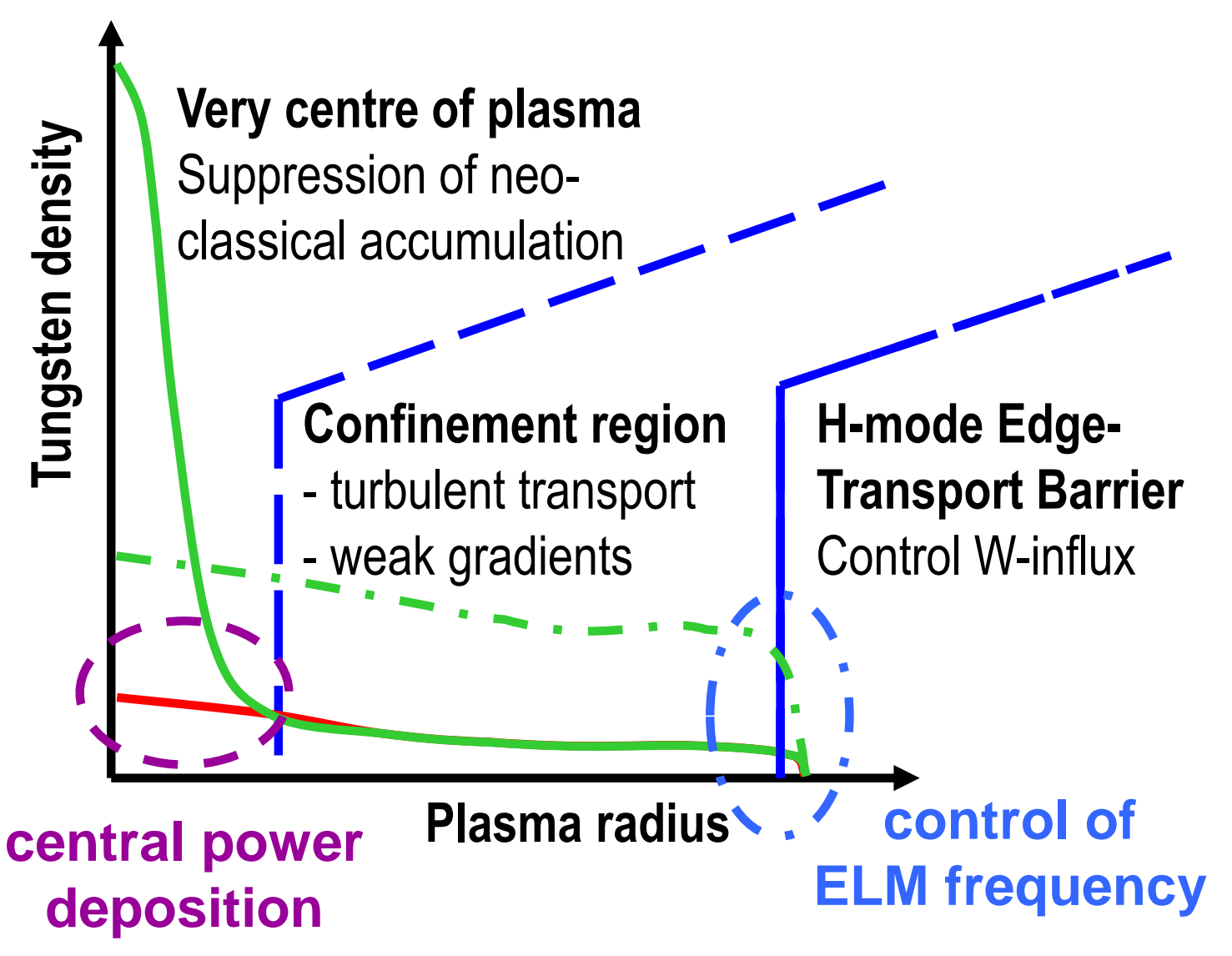




\section{ELM Pacing for W Flushing}

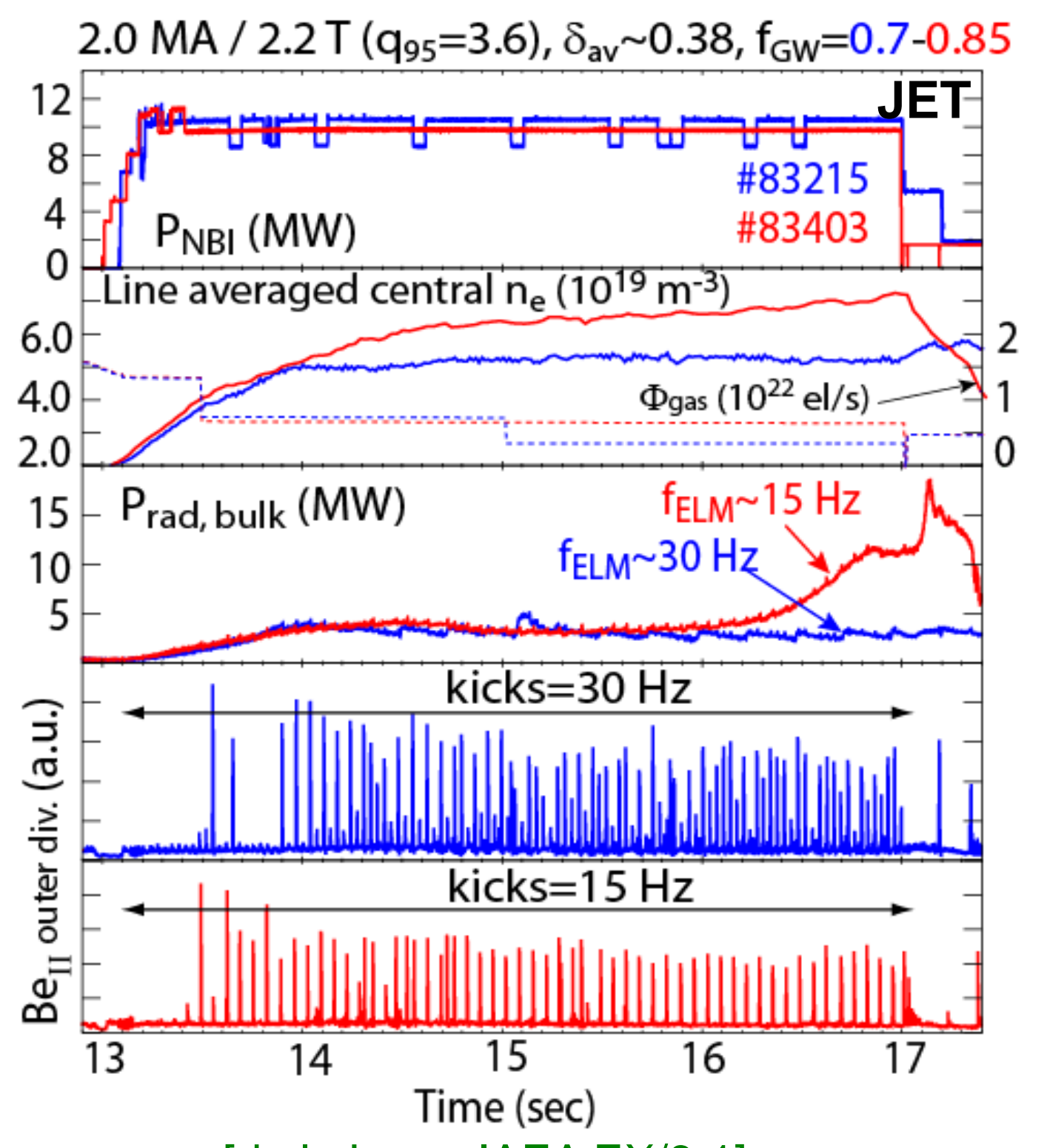

JET-ILW

- Vertical kicks: increase in $f_{E L M}$ reduces $W$ accumulation in gas fuelled $\mathrm{H}$-mode plasmas

- Pellets: ELM frequency increased by factor 4.5 stabilises W content

\section{AUG}

- Pellets ELM pace-making successfully flushes $\mathrm{W}$

- ELM mitigation by magnetic perturbation compatible with $\mathrm{W}$ suppression

[de la Luna, IAEA EX/6-1] 
 \\ Efficient suppression of $\mathrm{W}$ accumulation by central heating}

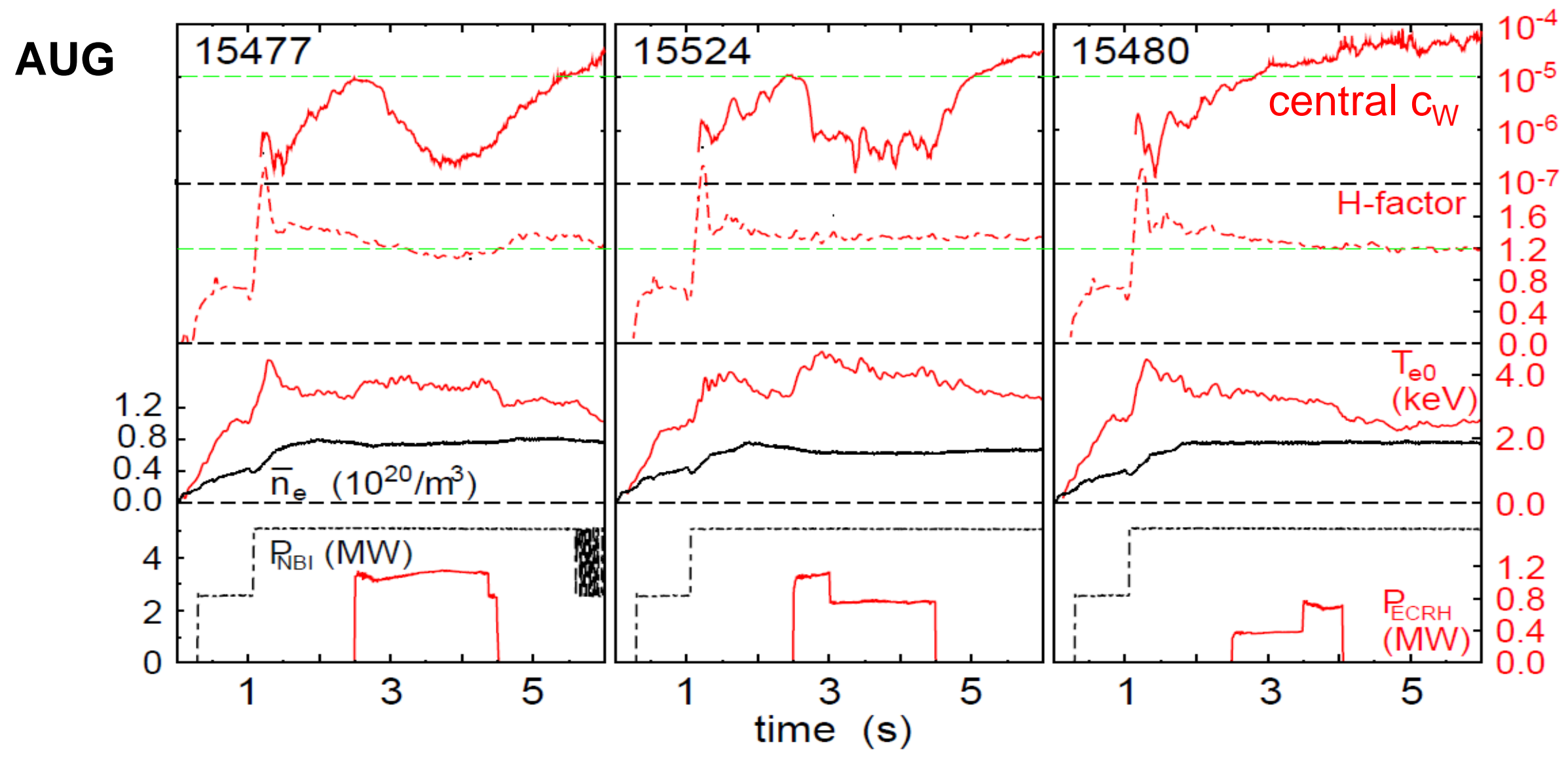

- tailoring of $\mathrm{ECRH}$ results in strong reduction of peaking of $\mathrm{C}_{\mathrm{W}}$ and moderate confinement degradation

- threshold for mitigation of peaking seems to depend on central radiation (transport reacts on local power balance?) 


\section{Non-disruptive $\mathrm{H}-\mathrm{L}$ cycle due to central radiation}

too large $\mathrm{W}$ influx and/or too low central transport can cause $\mathrm{W}$ accumulation

$\Rightarrow$ large central radiation

$\Rightarrow$ back transition to L-Mode

$\Rightarrow$ expulsion of $\mathrm{W}$

$\Rightarrow$ no disruption if heating is maintained

discharge can recover completely if $\mathrm{W}$ influx is reduced
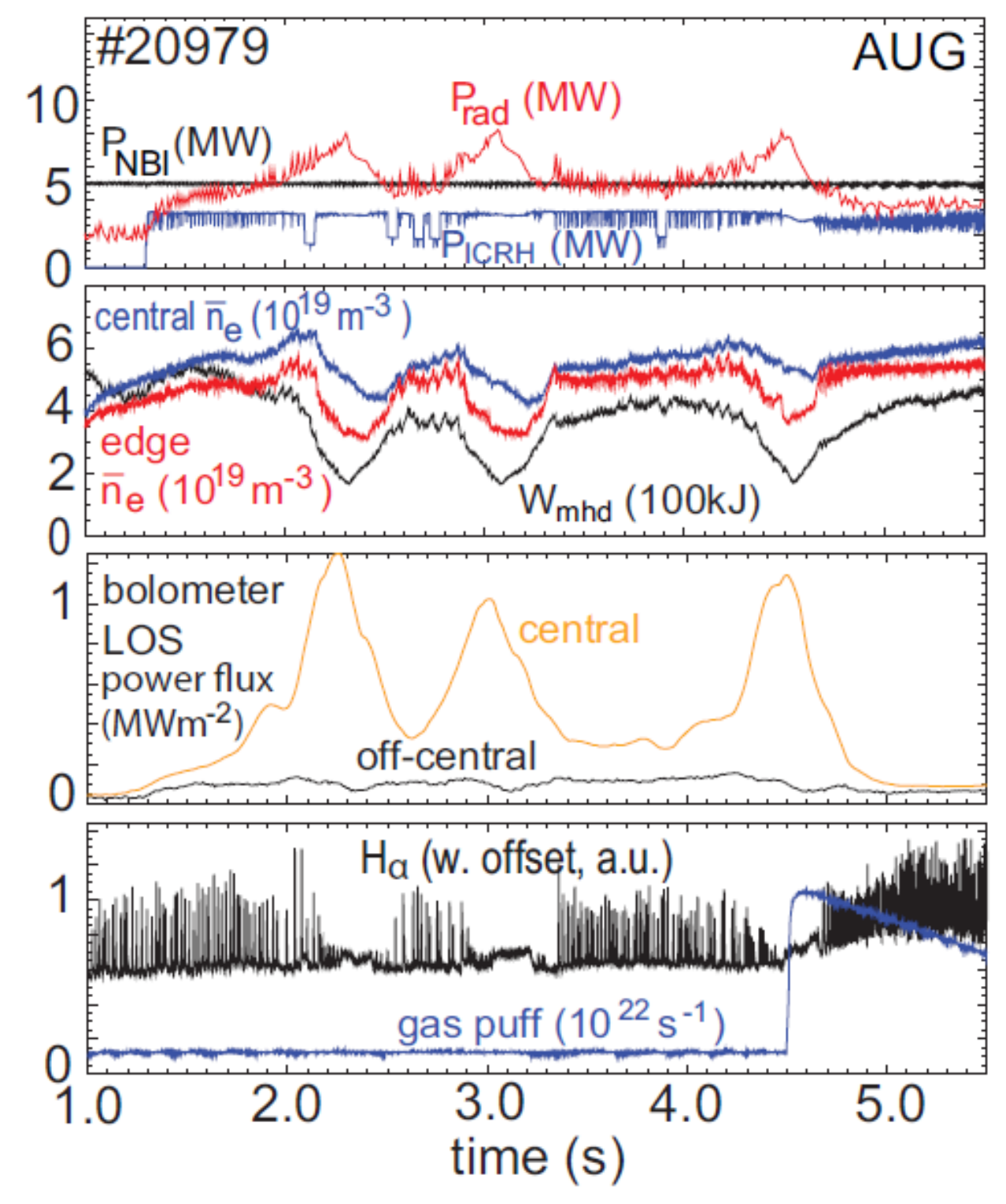


\section{LH Threshold Reduced by $25-30 \%$}

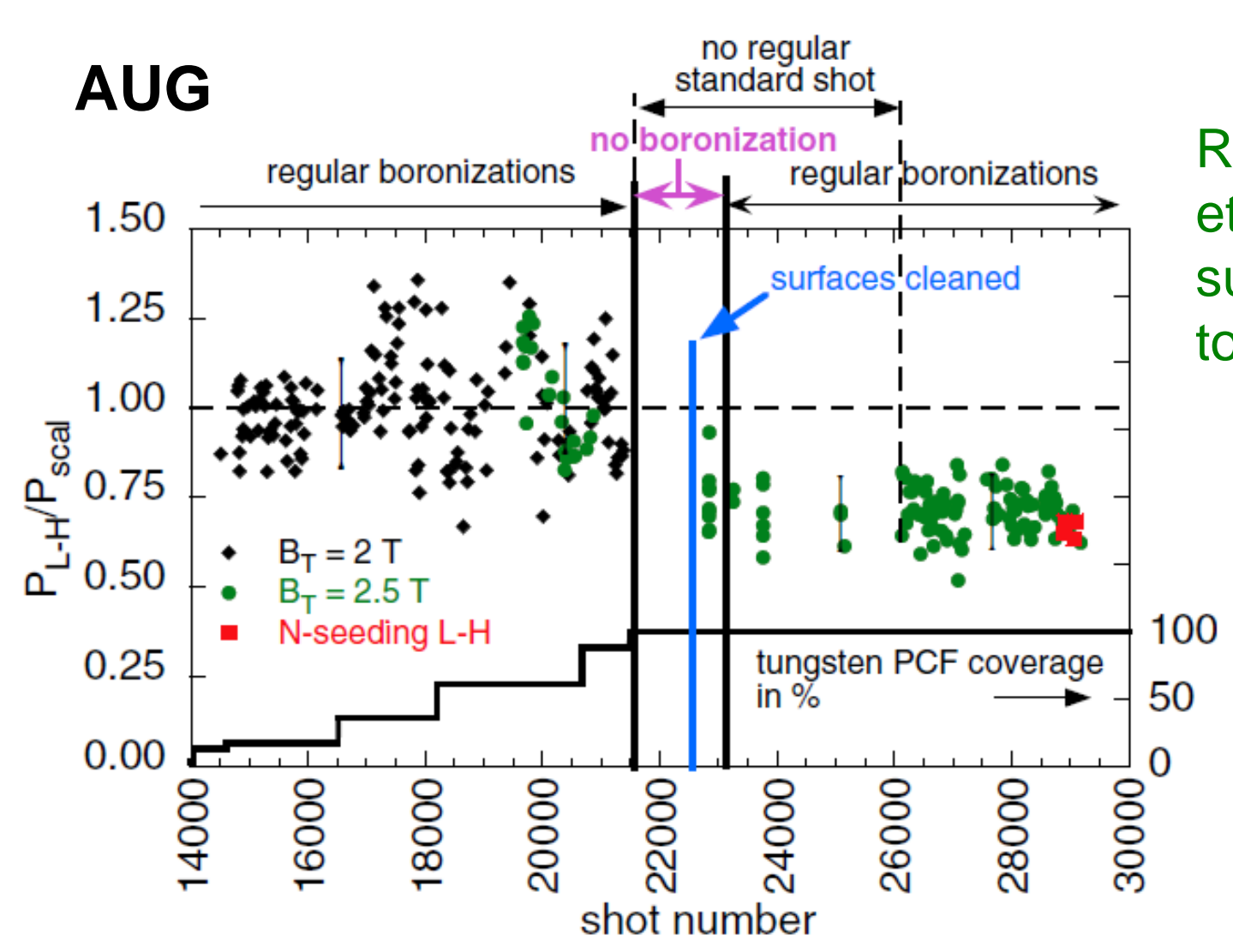

C dominated AUG/JET: $\mathrm{P}_{\text {th }} / \mathrm{P}_{\text {scal08 }} \approx 1$, AUG with full W PFCs: $P_{\text {th }} / P_{\text {scal08 }} \approx 0.75$ JET ILW:

$$
\mathrm{P}_{\text {th }} / \mathrm{P}_{\text {scal08 }} \approx 0.70
$$

Ryter et al., subm. to NF
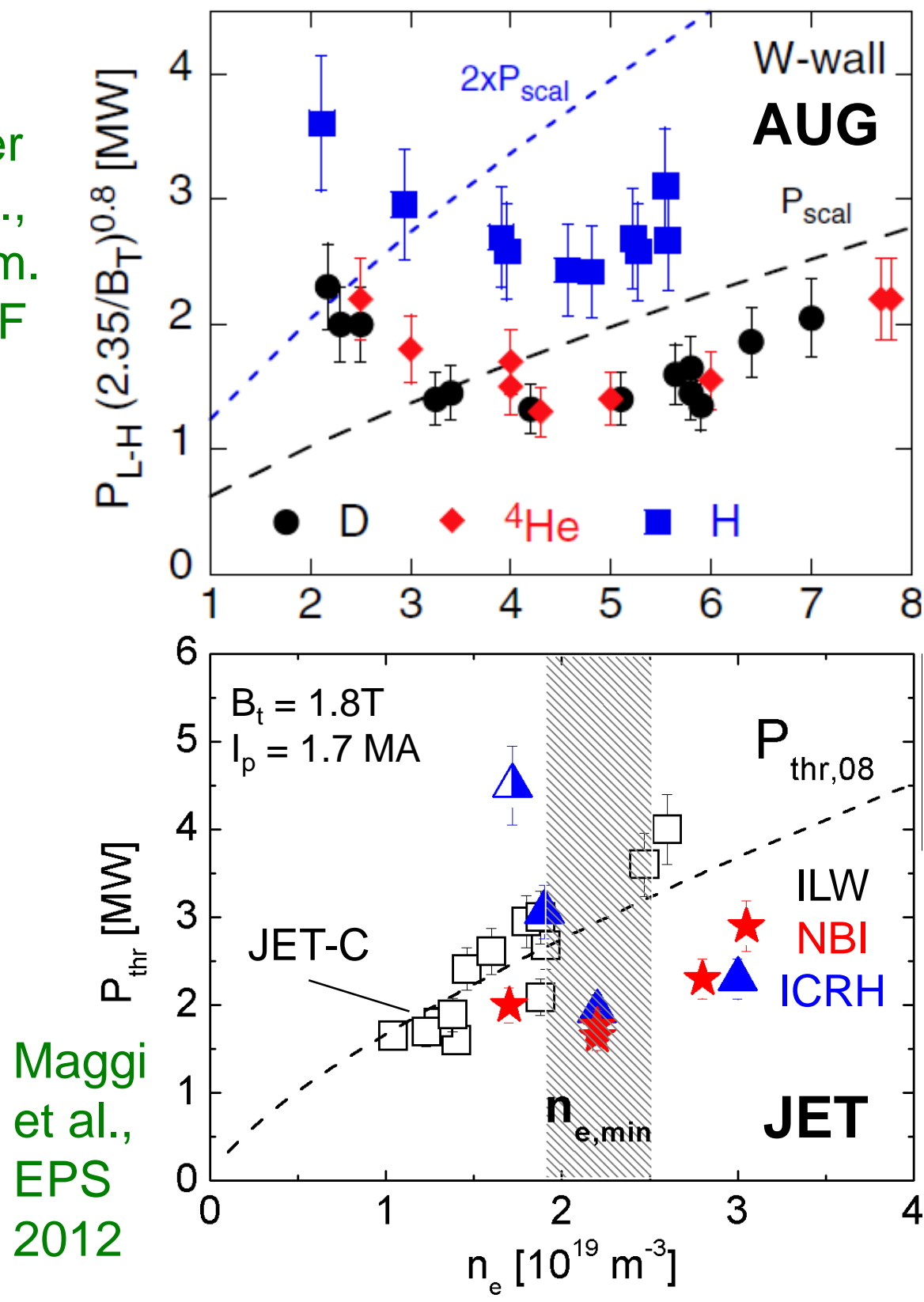


\section{Summary and Conclusions}

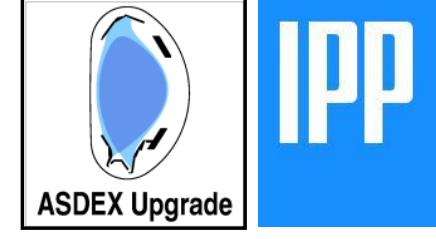

AUG and JET show very similar results concerning plasma

operation and W behavior

- facilitated conditioning $\Rightarrow$ highly beneficial during (ITER) operation

- low $C$ and $O$ content $\Rightarrow$ high plasma purity (low $Z_{\text {eff }}$ - Be plays major role in JET)

- large reduction of $D$ retention $\Rightarrow$ consistent with extrapolations to ITER

- significantly lower L-H threshold $\Rightarrow$ easier H-Mode access

But also

- strongly reduced radiation during disruptions $\Rightarrow$ mitigation necessary/relevant

- efficient ICRH but increased W influx $\Rightarrow$ important for antenna optimisation

- reduced operational space (no zero gas-puff!) $\Rightarrow$ focus on ITER relevant operation schemes

- observation of W accumulation $\Rightarrow$ confirmation/development of counter measures

- lower pedestal confinement (in JET) $\Rightarrow$ no effect of W radiation!

$\leftrightarrow$ new physics insights (correlation with edge radiation/dilution?) 